\title{
Prevalência de delirium em idosos submetidos a cirurgias com anestesia geral endovenosa
}

Prevalence of delirium in elderly underwent surgeries with intravenous general anesthesia

Prevalencia del delirio en ancianos sometidos a cirugía con anestesia general intravenosa

Niliane Mayrink Stopa SOTERO

Faculdade de Medicina, Universidade José do Rosário Vellano (Unifenas/Alfenas), 37130-000 Alfenas - MG, Brasil https://orcid.org/0000-0001-9370-9569

Cláudio Daniel CERDEIRA

Departamento de Bioquímica (DBq), Instituto de Ciências Biomédicas (ICB), Universidade Federal de Alfenas (UNIFAL-MG) 37130-001 Alfenas - MG, Brasil https://orcid.org/0000-0002-7242-8028

Sergio Luis Assis FERREIRA

Faculdade de Medicina, Universidade José do Rosário Vellano (Unifenas/Alfenas), 37130-000 Alfenas - MG, Brasil https://orcid.org/0000-0002-3938-0309

Roberto Salvador GUIMARÃES

Faculdade de Medicina, Universidade José do Rosário Vellano (Unifenas/Alfenas), 37130-000 Alfenas - MG, Brasil https://orcid.org/0000-0002-0451-2447

Roberta Bessa Veloso SILVA

Faculdade de Medicina, Universidade José do Rosário Vellano (Unifenas/Alfenas), 37130-000 Alfenas - MG, Brasil https://orcid.org/0000-0003-4794-5872

Gérsika Bitencourt SANTOS

Faculdade de Medicina, Universidade José do Rosário Vellano (Unifenas/Alfenas), 37130-000 Alfenas - MG, Brasil https://orcid.org/0000-0003-0849-2786

\section{Resumo}

Introdução: anestésicos gerais (AGs) endovenosos podem gerar complicações pós-operatórias, com destaque para o delirium, uma condição que atrasa a recuperação funcional do paciente e acarreta maior custo hospitalar. Objetivo: este estudo investigou a prevalência de delirium pós-anestésico/operatório (DPAO) em um hospital Universitário. Metodologia: estudo transversal no qual foram avaliados pacientes com idade acima de 60 anos, submetidos a diferentes tipos de cirurgias utilizando AGs. $O$ miniexame do estado mental (MEEM) foi replicado em três momentos (MMP, MM1 e MM3): durante o pré-operatório (D0), um dia após a cirurgia (D1) e três dias após a cirurgia (D3). Resultados: entre os 28 pacientes que concluíram o estudo (50\% para cada sexo e faixa etária mais frequente entre 60 e 69 anos [64\%]), a maior parte relatou possuir entre 1 a 4 anos de estudo (53\%), e os AGs mais utilizados em ordem decrescente foram: o fentanil $(85,7 \%)$, remifentamil $(78,6 \%)$, propofol $(64,3 \%)$ e etomidato $(50 \%)$. A prevalência de DPAO foi $21,4 \%(n=6)$, com 14,3\% ( $n=4)$ dos casos ocorrendo em D1, 3,6\% $(n=1)$ em D3 e 3,6\% $(n=1)$ em ambos os períodos, havendo maior prevalência em cirurgias cardíacas. Houve significativa $(p<0,05)$ maior pontuação média para o sexo masculino. Não foi observada diferença entre o nível de escolaridade, faixa etária, tipo de cirurgia ou uso de AGs e a pontuação no MEEM. Conclusão: a prevalência de DPAO em idosos mostrou-se significativa e apenas a variável "sexo" influenciou na média de pontuação no MEEM.

Descritores: Anestesia Geral; Delírio; Assistência a Idosos.

\section{Abstract}

Introduction: intravenous general anesthetics (IGAs) can lead to postoperative complications, such as delirium, delaying patient's functional recovery and entails higher hospital costs. Objective: this study investigated the prevalence of postoperative delirium (POD) at a University Hospital. Methodology: a cross-sectional study in which was evaluated patients aged over 60-year-old who underwent different types of surgeries using IGA. The mini-mental state examination (MMSE) was replicated at three different moments (MMP, MM1 and MM3): during the preoperative period (D0), one day after surgery (D1) and three days after surgery (D3). Results: among the 28 patients who completed the study (50\% for each sex and the most frequent age group between 60 and 69 years [64\%]), most reported having between 1 and 4 years of study (53\%), and the most used IGAs in decreasing order were: fentanyl (85.7\%), remifentamil (78.6\%), propofol $(64.3 \%)$, and etomidate $(50 \%)$. The prevalence of POD was $21.4 \%(n=6)$, with $14.3 \%(n=4)$ of cases occurring in D1, 3.6\% $(n=1)$ in D3, and 3.6\% $(n=1)$ in both moments, with a higher prevalence in cardiac surgeries. There was a significant $(p<0.05)$ higher mean score for males in the three MMSE applications. No significant difference was observed between level of education, age, type of surgery or use of IGAs and the MMSE score. Conclusion: the prevalence of POD in a sample of elderly was significant, and only the variable "sex" influenced the mean MMSE score.

Descriptors: Anesthesia, General; Delirium; Old Age Assistance.

\section{Resumen}

Introducción: los anestésicos generales (AG) intravenosos pueden generar complicaciones posoperatorias, especialmente delirio, condición que retrasa la recuperación funcional del paciente y ocasiona un mayor costo hospitalario. Objetivo: este estudio investigó la prevalencia de delirio postanestésico/operatorio (DPAO) en un hospital universitario. Metodología: estudio transversal en el que se evaluaron pacientes mayores de 60 años que fueron sometidos a diferentes tipos de cirugía mediante AG. El mini examen del estado mental (MEEM) se replicó en tres momentos (MMP, MM1 y MM3): durante el período preoperatorio (D0), un día después de la cirugía (D1) y tres días después de la cirugía (D3). Resultados: entre los 28 pacientes que completaron el estudio (50\% para cada sexo y el grupo de edad más frecuente entre 60 y 69 años [64\%]), la mayoría refirió tener entre 1 y 4 años de estudio (53\%), y los AG más utilizados en orden decreciente fueron: fentanilo $(85,7 \%)$, remifentamilo $(78,6 \%)$, propofol $(64,3 \%)$ y etomidato $(50 \%)$. La prevalencia de DPAO fue del 21,4\% $(n=6)$, con $14,3 \%(n=4)$ de los casos ocurriendo en D1, 3,6\% $(n=1)$ en D3 y $3,6 \%(n=1)$ en ambos períodos, con mayor prevalencia en cirugía cardíaca. Hubo una puntuación media significativamente más alta $(p<0,05)$ para los hombres. No hubo diferencia entre el nivel educativo, el grupo de edad, el tipo de cirugía o el uso de AG y la puntuación del MEEM. Conclusión: la prevalencia de DPAO en ancianos fue significativa y solo la variable "sexo" influyó en la puntuación media en el MEEM. Descriptores: Anestesia General; Delirio; Asistencia a los Ancianos.

INTRODUÇÃO

\section{Anestésicos gerais (AGs) endovenosos} podem gerar acúmulo plasmático nos idosos e intoxicação medicamentosa, desde que tais medicamentos dependem de biotransformação para serem eliminados, sendo este um processo metabólico que entra em declínio com o avanço da idade no idoso ${ }^{1}$. Desse modo, nos idosos ocorrem diversas alterações metabólicas que interferem na farmacocinética e farmacodinâmica dos anestésicos, predispondoos a uma maior susceptibilidade para complicações pós operatória ${ }^{2-4}$. Complicações neurológicas na recuperação pós-anestésica são muito comuns 
em idosos, ocorrendo com incidência variável em diversos tipos de cirurgia. A deterioração pós-operatória ocorre em até $36,8 \%$ dos pacientes cirúrgicos e pode ser classificada em duas categorias principais: o delirium pósanestésico/operatório (DPAO, chegando até $31 \%$ dos casos) e a disfunção neurocognitiva moderada (DCPO) ${ }^{5-7}$.

O DPAO é um distúrbio psiquiátrico agudo, sendo o mais comum em pacientes idosos hospitalizados. Basicamente, os indícios que caracterizam o DPAO são: déficit de consciência; alteração na cognição ou distúrbio de percepção de surgimento imediato; desenvolvimento do delirium em curto espaço de tempo (horas ou dias), com flutuações durante o curso do dia e evidências de uma possível causa (como o uso de AGs). O delirium no paciente idoso pode se manifestar com quadros de torpor, sonolência, desatenção e letargia $^{8}$. Ao passo que a DCPO acarreta prejuízo na memória, concentração, compreensão da linguagem e integração social, e possui maior incidência em pacientes idosos submetidos a cirurgias ${ }^{5}$.

Complicações na evolução de aproximadamente $35 \%$ dos pacientes cirúrgicos são causadas por DPAO ou DCPO, sendo relacionada com períodos prolongados das internações de pacientes idosos no pósoperatório, pior recuperação funcional após a alta e maiores custos para o paciente e hospital. A mortalidade associada ao DPAO é $13 \%$ e aproximadamente $72 \%$ dos pacientes idosos acometidos morrem dentro de cinco anos após o procedimento cirúrgico, comparado com $34,7 \%$ dos pacientes que permanecem lúcidos no pós-operatório ${ }^{9}$. Portanto, a escolha do agente anestésico ou a combinação apropriada é fundamental para aliviar estímulos nocivos, estresse e ansiedade, ao mesmo tempo em que minimiza o risco de eventos adversos ${ }^{10}$.

Neste contexto, torna-se necessário uma melhor avaliação do paciente no pré e pósoperatório, para o diagnóstico e tratamento precoce dessas disfunções. Cerca de $70 \%$ dos pacientes idosos com DPAO não são diagnosticados e a detecção precoce beneficia a prevenção, recuperação e qualidade de vida do paciente ${ }^{8}$. Assim, o presente estudo avaliou a prevalência de DPAO após procedimentos cirúrgicos com AGs endovenosos em idosos atendidos em um hospital universitário do sul de Minas Gerais, Brasil.

MATERIAL E MÉTODO

Estudo transversal, envolvendo idosos submetidos a cirurgias com anestesia geral endovenosa, conduzido em um hospital universitário de médio porte do sul de Minas Gerais, Brasil, entre Março de 2016 e Junho de 2017, no qual se analisou prontuários dos pacientes voluntários e conduziu-se uma entrevista norteada por um questionário semiestruturado, elaborado pelos entrevistadore. Posteriormente, avaliou-se o estado mental com uso do mini-exame do estado mental (MEEM), com adequações de acordo com a literatura pertinente ${ }^{11}$. O estudo foi aprovado pelo Comitê de Ética e Pesquisa envolvendo humanos da Universidade José do Rosário Vellano (CAAE: 42983115.2.0000.5143; Parecer número: 1.014.861). Todos os pacientes voluntários foram informados e esclarecidos sobre 0 presente estudo verbalmente e por escrito e assinaram o Termo de Consentimento Livre e Esclarecido (TCLE).

Preliminarmente, os critérios de inclusão dos voluntários no estudo foram: ambos os sexos, submetidos a procedimentos cirúrgicos com AGs endovenosos, acima de 60 anos de idade. Como critérios de exclusão, não foram avaliados: os indivíduos fora da faixa etária; indivíduos que não aceitaram participar da pesquisa; indivíduos com alterações cognitivas incapazes de responder aos questionários; portadores de doenças psiquiátricas ou cognitivas prévias e casos em que algum evento adverso tenha ocorrido no período perianestésico ou pós-operatório imediato e aqueles pacientes submetidos a cirurgia neurológica.

Para avaliar o DPAO utilizou-se o MEEM, que permite avaliar sete categorias do estado mental nos pacientes voluntários: orientação temporal, orientação espacial, memória imediata, atenção e cálculo, memória de evocação, linguagem e capacidade construtiva visual ${ }^{11}$. O questionário foi aplicado (estatística kappa para o grau de concordância entre os entrevistadores $>0,8)$ em três momentos: durante o pré-operatório (D0), um dia após a cirurgia (D1) e três dias após a cirurgia (D3). Portanto, os pacientes foram submetidos a uma primeira aplicação do MEEM no pré-operatório (MMP) em D0 e, após o procedimento cirúrgico, em dois tempos: no pós-operatório de um dia (D1), com a primeira reaplicação (MM1) e no pós-operatório de três dias (D3), com a segunda reaplicação (MM2). A pontuação no MEEM foi reportada como: pré (MMP), 1 dia (MM1) e 3 dias após a cirurgia (MM2). A pontuação máxima é 30 pontos, sendo considerado um déficit cognitivo nas seguintes condições: pacientes não alfabetizados com resultado menor ou igual a 15 pontos; pacientes de 1 a 11 anos de escolaridade com pontuação menor ou igual a 
22; pacientes com escolaridade superior a 11 anos com pontuação igual ou menor que 27 pontos $^{11}$. Portanto, na interpretação individual do MEEM, foram considerados casos de DPAO em D1 e/ou D3 quando as pontuações do MM1 e/ou MM2 foram menores ou iguais para a faixa de escolaridade supracitada e menor que o respectivo controle (D0).

A análise de variância (ANOVA) foi executada no software $R$ ( $R$ CORE TEAM, 2017) para comparar as médias provenientes da estratificação dos voluntários de acordo com o protocolo de AGs, cirurgia realizada, o sexo, faixa etária e escolaridade. Os intervalos de confiança de $95 \%\left(\mathrm{IC}_{(95 \%)}\right)$ foram construídos no software BioEstat ${ }^{\circledR} 5.0$ (Belém/PA, Brasil, 2007). RESULTADOS

Após a análise dos prontuários, foram selecionados 36 voluntários, dos quais 28 concluíram o estudo. Da casuística avaliada, houve equilíbrio entre os sexos (50\% vs. $50 \%)$, com faixa etária predominante entre 60 e 69 anos, seguidos de $29 \%$ com idade entre 70 e 79 anos e, apenas $7 \%$ na faixa de 80 a 89 anos. A maioria $(53,57 \%)$ relatou possuir entre 1 a 4 anos de estudo. Os dados sociodemográficos são apresentados na Tabela 1.

Tabela 1. Dados sociodemográficos na amostra de pacientes submetidos a procedimentos cirúrgicos com anestesia geral endovenosa em um hospital universitário no sul de Minas Gerais, Brasil

\begin{tabular}{|c|c|c|c|}
\hline \multirow{2}{*}{\multicolumn{2}{|c|}{ Variáveis }} & \multicolumn{2}{|c|}{ Resultados entre os 28 pacientes } \\
\hline & & $n(\%)$ & IC95(\%) \\
\hline \multirow{3}{*}{ Sexo } & Masculino & $14(50 \%)$ & $31,5-68,5$ \\
\hline & Feminino & $14(50 \%)$ & $31,5-68,5$ \\
\hline & Total & $28(100 \%)$ & \\
\hline \multirow{4}{*}{ Faixa etária (anos) } & $60-69$ & $18(64 \%)$ & $46,5-82$ \\
\hline & $70-79$ & $8(29 \%)$ & $11,8-45,3$ \\
\hline & $80-89$ & $2(7 \%)$ & ---- \\
\hline & Total & $28(100 \%)$ & \\
\hline \multirow{6}{*}{$\begin{array}{l}\text { Escolaridade } \\
\text { (anos de estudo) }\end{array}$} & $>11$ & $3(11 \%)$ & ---- \\
\hline & $8-11$ & $5(18 \%)$ & ---- \\
\hline & 5-7 & $4(14 \%)$ & ---- \\
\hline & $1-4$ & $15(53 \%)$ & $35,1-72$ \\
\hline & Não alfabetizado & $1(4 \%)$ & --- \\
\hline & Total & $28(100 \%)$ & \\
\hline
\end{tabular}

Após aplicação prévia de questionário e avaliação inicial do MEEM, os pacientes foram submetidos a diferentes protocolos analgésicoanestésicos e as respectivas cirurgias, como apresentado nas Figuras 1 a 6 . Observou-se que os analgésicos/anestésicos mais utilizados foram o fentanil $(85,7 \%)$, remifentanil $(78,6 \%)$, propofol $(64,3 \%)$ e etomidato (50\%). A cirurgia de revascularização miocárdica (CRVM) foi a mais frequente entre as realizadas $(46,4 \%)$, seguida da colescistectomia por vídeo (CCV, $21,4 \%$ ), a troca de valva mitral (CTVM, 10,7\%) e artrodese coluna lombar (CACL, 7,14\%), cujos protocolos analgésico-anestésicos são mostrados em detalhes nas Figuras 1 e 2.

Avaliando-se especificamente os protocolos analgésico-anestésicos adotados conforme o tipo de procedimento cirúrgico
(Figuras 3 a 6), as combinações mais frequentes foram: na CRVM fentanil, etomidato e remifentanil (54\%, sete vezes maior quando comparado aos demais tipos de combinações); na CCV fentanil, propofol e remifentanil (33\%) e fentanil e propofol (33\%); na CACL fentanil, etomidato, remifentanil (50\%) e fentanil, propofol, remifentanil (50\%); e finalmente, na CTVM fentanil, propofol, remifentanil e "sevoflurano" $(33,3 \%)$ e fentanil, propofol, remifentanil e "isoflurano" (33,3\%) fentanil, etomidato, remifentanil $(33,3 \%)$.

Dos 36 voluntários previamente selecionados, dois vieram a óbito durante cirurgia, quatro tiveram alta hospitalar antes da realização do questionário $\mathrm{D} 1$ e dois pacientes não quiseram responder os questionários $\mathrm{D} 1 \mathrm{e}$ D3, portanto, explicando os 28 voluntários que participaram do MEEM, objeto de estudo. Entre os 28 pacientes, dez pacientes realizaram apenas 0 primeiro questionário pós-cirúrgico (D1), que correspondem aos que realizaram cirurgias de internação rápida, como CCV. Dois pacientes não conseguiram responder ao primeiro questionário pós-cirúrgico (D1), pois tiveram evolução clínica prolongada no pósoperatório, sendo que os dois realizaram CTVM. $\mathrm{Na}$ Tabela 2 é apresentada a avaliação individual do MEEM, revelando uma prevalência total de DPAO como $21,4 \%(n=6)$, sendo que $14,3 \%(n=4)$ dos casos ocorreram em D1, $3,6 \%(n=1)$ em D3 e $3,6 \%(n=1)$ em ambos os períodos.

No geral, observou-se que a variável sexo apresentou diferença significativa na pontuação no MEEM $(p<0,05)$. O sexo masculino apresentou maior pontuação média de 26,5; 25,4; e 26,9 respectivamente, nos MMP, MM1 e MM2, comparado ao feminino, com: 25,4; 25,5; e 24,6, respectivamente (Tabela 3).

A análise da interação entre o nível de escolaridade ou faixa etária e a pontuação no MEEM não revelou interação significativa, ou seja, independentemente do nível de escolaridade ou faixa etária, a pontuação média pode ser considerada a mesma $(p=0,90)$ (Tabela 3). Observou-se alta precisão das estimativas (CV $=13,7 \%)$. $\mathrm{Na}$ avaliação da interação entre os tipos de protocolos analgésico-anestésicos ou de cirurgia e a pontuação no MEEM (Tabela 4), os dados revelaram que não houve significância estatística $(p=0,67)$. A variabilidade também foi baixa $(\mathrm{CV}=14,4 \%)$. 
Tabela 2. Interpretação individual do miniexame do estado mental (MEEM), para determinação da prevalência de delirium pós-anestésico/operatório (DPAO) entre os 28 pacientes submetidos a procedimentos cirúrgicos com anestesia geral endovenosa em um hospital universitário no sul de Minas Gerais, Brasil.

\begin{tabular}{|c|c|c|c|c|c|c|c|c|}
\hline Sexo & Idade & EC & PC & PAA & MMP & MM1 & MM2 & INTP \\
\hline $\mathrm{F}$ & $60-69$ & 1-4 anos & CRVM & $\begin{array}{l}\text { fentanil, } \\
\text { etomidato, } \\
\text { remifentanil }\end{array}$ & 21 & 23 & 22 & Normal \\
\hline M & 70-79 & 5-7 anos & CRVM & $\begin{array}{l}\text { fentanil, } \\
\text { etomidato, } \\
\text { remifentanil }\end{array}$ & 26 & 20 & 23 & $\begin{array}{l}\text { DPAO } \\
\text { (D1) }\end{array}$ \\
\hline $\mathrm{F}$ & $60-69$ & 5-7 anos & CRVM & $\begin{array}{l}\text { fentanil, } \\
\text { etomidato, } \\
\text { remifentanil }\end{array}$ & 28 & 26 & 27 & Normal \\
\hline M & $60-69$ & 8-11 anos & CACL & $\begin{array}{l}\text { fentanil, etomidato, } \\
\text { remifentanil }\end{array}$ & 28 & 27 & NA & Normal \\
\hline $\mathrm{F}$ & $60-69$ & 1-4 anos & CRVM & $\begin{array}{l}\text { fentanil, etomidato, } \\
\text { remifentanil }\end{array}$ & 24 & 22 & 22 & $\begin{array}{l}\text { DPAO } \\
(\text { D1 e D3) }\end{array}$ \\
\hline м & $60-69$ & $\geq 11$ anos & CRVM & $\begin{array}{l}\text { fentanil, } \\
\text { etomidato, } \\
\text { remifentanil }\end{array}$ & 30 & 30 & 29 & Normal \\
\hline M & $60-69$ & 1-4 anos & CRVM & $\begin{array}{l}\text { fentanil, } \\
\text { etomidato, } \\
\text { remifentanil }\end{array}$ & 30 & 30 & 30 & Normal \\
\hline M & 70-79 & 1-4 anos & CRVM & $\begin{array}{l}\text { remifentanil, } \\
\text { etomidato, } \\
\text { propofol }\end{array}$ & 29 & 27 & 26 & Normal \\
\hline $\mathrm{M}$ & $60-69$ & 1-4 anos & CRVM & $\begin{array}{l}\text { remifentanil, } \\
\text { etomidato }\end{array}$ & 27 & 25 & 23 & Normal \\
\hline M & $60-69$ & 1-4 anos & CRVM & $\begin{array}{l}\text { fentanil, } \\
\text { propofol, } \\
\text { remifentanil }\end{array}$ & 30 & 26 & 28 & Normal \\
\hline $\mathrm{F}$ & $60-69$ & 8-11 anos & CTVM & $\begin{array}{l}\text { fentanil, } \\
\text { propofol, } \\
\text { remifentanil }\end{array}$ & 26 & 25 & NA & Normal \\
\hline $\mathrm{F}$ & 70-79 & $1-4$ anos & $\mathrm{CCV}$ & $\begin{array}{l}\text { fentanil, } \\
\text { propofol, } \\
\text { etomidato }\end{array}$ & 23 & 20 & AA & $\begin{array}{l}\text { DPAO } \\
\text { (D1) }\end{array}$ \\
\hline $\mathrm{F}$ & $60-69$ & $\geq 11$ anos & CRVM & $\begin{array}{l}\text { fentanil, } \\
\text { etomidato } \\
\text { remifentanil, } \\
\text { propofol }\end{array}$ & 29 & 29 & 30 & Normal \\
\hline $\mathrm{F}$ & $60-69$ & 8-11 anos & CACL & $\begin{array}{l}\text { fentanil, } \\
\text { propofol, } \\
\text { remifentanil }\end{array}$ & 26 & 27 & $\mathrm{AA}$ & Normal \\
\hline $\mathrm{M}$ & $60-69$ & 8-11 anos & CRVM & $\begin{array}{l}\text { remifentanil, } \\
\text { propofol }\end{array}$ & 30 & 28 & 29 & Normal \\
\hline M & 70-79 & 1-4 anos & CTVA & $\begin{array}{l}\text { fentanil, } \\
\text { remifentanil, } \\
\text { propofol }\end{array}$ & 27 & 25 & 28 & Normal \\
\hline $\mathrm{F}$ & $80-89$ & 1-4 anos & CRVM & $\begin{array}{l}\text { fentanil, } \\
\text { etomidato, } \\
\text { remifentanil }\end{array}$ & 29 & 29 & 29 & Normal \\
\hline M & 70-79 & 1-4 anos & $\mathrm{CCV}$ & $\begin{array}{l}\text { fentanil, } \\
\text { propofol, } \\
\text { remifentanil }\end{array}$ & 27 & 25 & $\mathrm{AA}$ & Normal \\
\hline M & $60-69$ & 5-7 anos & CRVM & $\begin{array}{l}\text { fentanil, } \\
\text { etomidato, } \\
\text { propofol }\end{array}$ & 30 & 28 & 30 & Normal \\
\hline $\mathrm{F}$ & $80-89$ & $<1$ ano & $\mathrm{CCV}$ & $\begin{array}{l}\text { fentanil, } \\
\text { propofol, } \\
\text { remifentanil }\end{array}$ & 27 & 26 & $\mathrm{AA}$ & Normal \\
\hline $\mathrm{F}$ & $60-69$ & 1-4 anos & $\mathrm{CCV}$ & Propofol, remifentanil & 21 & 22 & $\mathrm{AA}$ & Normal \\
\hline $\mathrm{M}$ & $70-79$ & 1-4 anos & $\mathrm{CCV}$ & fentanil, propofol & 19 & 24 & 23 & Normal \\
\hline $\mathrm{F}$ & $60-69$ & 1-4 anos & CTTEC & fentanil, propofil & 23 & 24 & AA & Normal \\
\hline $\mathrm{F}$ & 70-79 & 5-7 anos & $\mathrm{CCV}$ & $\begin{array}{l}\text { fentanil, } \\
\text { propofol }\end{array}$ & 28 & 21 & $\mathrm{AA}$ & $\begin{array}{l}\text { DPAO } \\
\text { (D1) }\end{array}$ \\
\hline M & $60-69$ & 1-4 anos & CRCF & $\begin{array}{l}\text { fentanil, } \\
\text { propofol, } \\
\text { remifenta }\end{array}$ & 18 & 18 & $\mathrm{AA}$ & Normal \\
\hline M & $60-69$ & $\geq 11$ anos & CBAEF & $\begin{array}{l}\text { fentanil, } \\
\text { propofol }\end{array}$ & 25 & 23 & AA & $\begin{array}{l}\text { DPAO } \\
\text { (D1) }\end{array}$ \\
\hline $\mathrm{F}$ & 70-79 & 1-4 anos & CTVM & $\begin{array}{l}\text { Fentanil, } \\
\text { propofol, } \\
\text { remifentanil }\end{array}$ & 22 & I & 17 & $\begin{array}{l}\text { DPAO } \\
\text { (D3) }\end{array}$ \\
\hline $\mathrm{F}$ & $60-69$ & 8-11 anos & CTVM & \begin{tabular}{|l|} 
fentanil, \\
etomidato, \\
remifentanil
\end{tabular} & 28 & I & 25 & Normal \\
\hline
\end{tabular}

Legenda: $\mathrm{EC}=$ Escolaridade (anos de estudo);

$\mathrm{PC}=$ Procedimento Cirúrgico; CRVM, cirurgia de revascularização miocárdica; CACL, cirurgia de artrodese de coluna lombar; CTVM, cirurgia de troca de valva mitral; CCV, cirurgia de colescistectomia por vídeo; CTVA, cirurgia de troca de valva aórtica; CTTEC, cirurgia de tireoidectomia total + esvaziamento cervical; CRCF, cirurgia de reconstrução crânio-facial; CBAEF, cirurgia bypass aortofemoral endarterect femoropoplitea;

I, Intubado; AA, Alta antes; NA, não aderiu.

INTP = Interpretação

DPAO foi estabelecida de acordo com Brucki et al. ${ }^{11}$

Paciente não alfabetizado ( $<1$ ano de estudo) com pontuação menor ou igual a 15

Paciente com 1 a 11 anos de estudo com pontuação menor ou igual a 22

Paciente com escolaridade superior a 11 anos e pontuação igual ou menor que 27

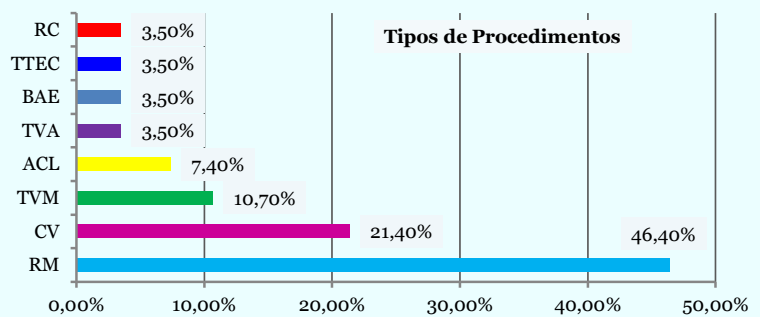

Figura 1. Frequência dos tipos de procedimentos cirúrgicos na amostra de pacientes entrevistados em um hospital universitário no sul de Minas Gerais, Brasil. (Fonte: Dados da Pesquisa).

Legenda: $\mathrm{RC}=$ Reconstrução Craniofacial:

TTEC+ Tireoidectomia Total + Esvaziamento Cervical;

$\mathrm{BAE}=$ Bypass Aortofemoral Endarterectomia;

TVA = Troca de Valva Aórtica;
ACL Artrodese de Coluna Lombar

TVM = Troca de Valva Mitral:

TVM = Troca de Valva Mitral;
$\mathrm{CV}=$ Colecistectomia Videolaparoscópica;

$\mathrm{RV}=$ Revascularização do miocárdio

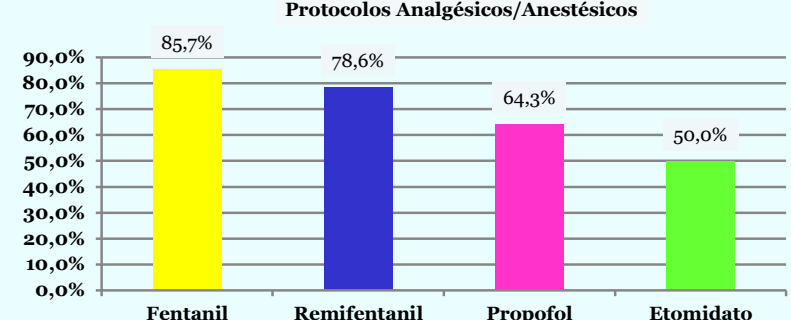

Figura 2. Frequências dos protocolos analgésicos/anestésicos (anestesia geral endovenosa) adotados na amostra de pacientes entrevistados em um hospital universitário no sul de Minas Gerais, Brasil. (Fonte: Dados da Pesquisa).

\section{Protocolos Analgésicos/Anestésicos em CRVM}

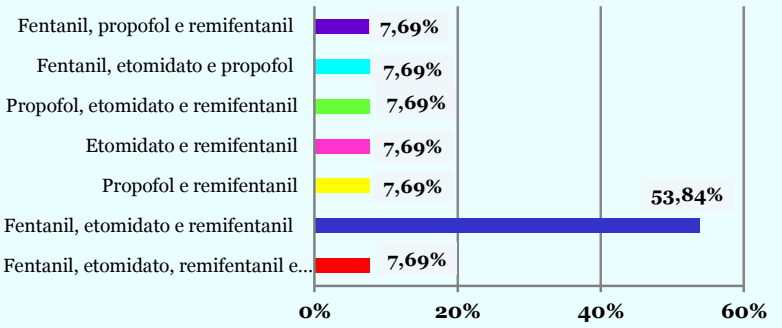

Figura 3. Frequências percentuais dos protocolos analgésico-anestésicos adotados em cirurgias de revascularização miocárdica (CRVM) na amostra de pacientes em hospital universitário no sul de Minas Gerais, Brasil (Fonte: dados da Pesquisa).

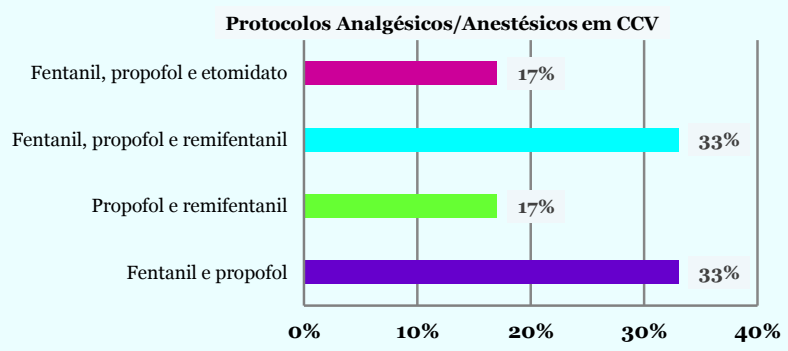

Figura 4. Frequências percentuais dos protocolos analgésico-anestésicos adotados em cirurgias de colecistectomia por vídeo (CCV) na amostra de pacientes em hospital universitário no sul de Minas Gerais, Brasil (Fonte: dados da Pesquisa).

Protocolos Analgésicos/Anestésicos em CACL

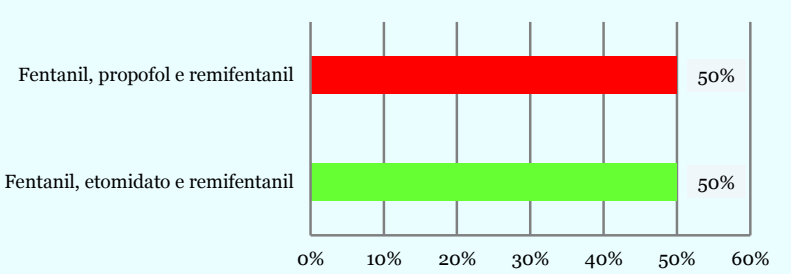

Figura 5. Frequências percentuais dos protocolos analgésico-anestésicos adotados em cirurgias de artrodese de coluna lombar (CACL) na amostra de pacientes em hospital universitário no sul de Minas Gerais, Brasil (Fonte: dados da Pesquisa).

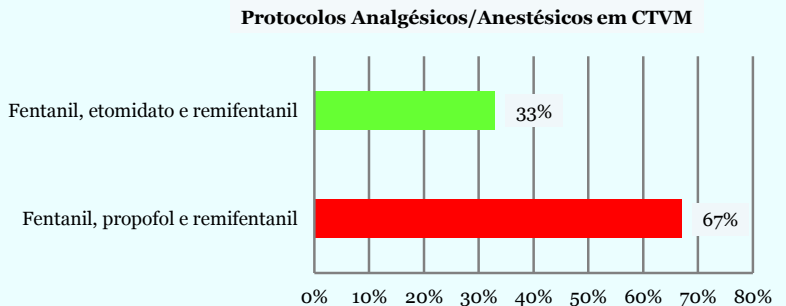

Figura 6. Frequências percentuais dos protocolos analgésico-anestésicos adotados em cirurgias de troca de valva mitral (CTVM) na amostra de pacientes em hospital universitário no sul de Minas Gerais, Brasil (Fonte: dados da Pesquisa). 
Tabela 3. Pontuação geral no miniexame do estado mental (MEEM) estratificada por variáveis sociodemográficas, entre os 28 pacientes submetidos a procedimentos cirúrgicos com anestesia geral endovenosa em um hospital universitário no sul de Minas Gerais, Brasil.

\begin{tabular}{c|c|c|c|c|c|}
\hline TMM & $0-12$ & $13-17$ & $\mathbf{1 8 - 2 2}$ & $\mathbf{2 3}-\mathbf{3 0}$ & MEDIA
\end{tabular}

\begin{tabular}{|l|c} 
Variável - Escolaridade (Anos de Estudo) & Total de Participantes ${ }^{A}$ \\
\hline
\end{tabular}

\begin{tabular}{l|c|c|c|c|c|c} 
TESTE & \multicolumn{4}{|c|}{ Analfabetos (1 ou menos anos de estudo) } & (1) & \\
\hline MMP & 0 & 0 & 0 & 1 & 1 & 27 \\
\hline MM1 & 0 & 0 & 0 & 1 & 1 & 26 \\
\hline
\end{tabular}

MM1

MM2** $^{\text {TESTE }}$

MMP

$\mathrm{MM1}^{*}$

MM2***

MMP

$\mathrm{MM}^{* *}$

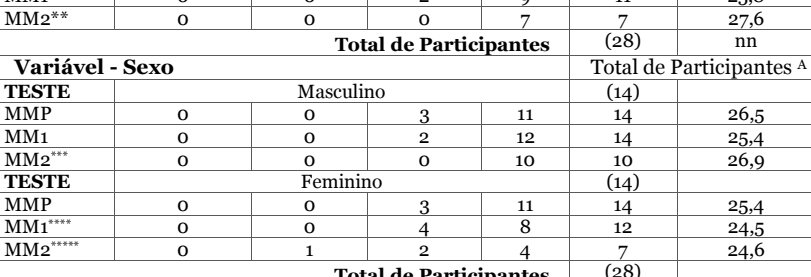

Total de Participantes $\quad(28)$

${ }^{\text {A }}$ para a variável (entre parênteses) e em cada fase do teste; 2 pacientes estavam entubados e incapazes de responder ao questionário; "* 11 pacientes tiveram alta antes da terceira aplicação do MEEM ou recusaramse a responder o teste; 4 pacientes tiveram alta antes da terceira reaplicação do MEEM ou recusaram-se a responder o teste; ${ }_{*+* *+*}^{* *} 2$ pacientes estavam intubados incapazes de responder o questionário; ${ }^{* \star * *} 7$ pacientes tiveram alta antes da terceira reaplicação do MEEM ou recusaram-se a responder o teste.

Tabela 4. Pontuação geral no miniexame do estado mental (MEEM) estratificada por tipos de protocolos analgésicos/anestésicos adotados entre os 28 pacientes submetidos a procedimentos cirúrgicos com anestesia geral endovenosa, em um hospital universitário no sul de Minas Gerais, Brasil.

\begin{tabular}{|c|c|c|c|c|c|c|}
\hline TMM & 0-12 & 13-17 & 18-22 & 23-30 & & Média \\
\hline Variável & \multicolumn{4}{|c|}{ Agente Anestésico/ Analgésico } & \multicolumn{2}{|c|}{$\mathrm{N}^{0}$ Participantes } \\
\hline MMP & 0 & o & 1 & 8 & 10 & 27,1 \\
\hline MM1* & 0 & 0 & 2 & 6 & 8 & 25,9 \\
\hline $\mathrm{MM}^{* * *}$ & 0 & 0 & 2 & 6 & 8 & 25,9 \\
\hline \multicolumn{5}{|c|}{ Teste - Remifentanil, etomidato, propofol } & (1) & $\mathrm{M}$ \\
\hline MMP & 0 & 0 & 0 & 1 & 1 & 29 \\
\hline MM1 & 0 & 0 & 0 & 1 & 1 & 27 \\
\hline MM2 & 0 & o & 0 & 1 & 1 & 26 \\
\hline \multicolumn{5}{|c|}{ Teste - Fentanil, propofol, remifentanil } & (8) & \\
\hline MMP & 0 & 0 & 2 & 6 & 8 & 25,4 \\
\hline MM1 & o & o & 1 & 6 & 7 & 24,6 \\
\hline MM2 & 0 & o & 1 & 1 & 2 & 24,3 \\
\hline \multicolumn{5}{|c|}{ Teste - Remifentanil, etomidato } & (1) & \\
\hline MMP & 0 & 0 & 0 & 1 & 1 & 27 \\
\hline MM1 & 0 & o & o & 1 & 1 & 25 \\
\hline MM2 & 0 & 0 & 0 & 1 & 1 & 23 \\
\hline \multicolumn{5}{|c|}{ Teste - Fentanil, propofol, etomidato } & (2) & \\
\hline MMP & 0 & 0 & 3 & 11 & 14 & 26,5 \\
\hline MM1 & o & o & 4 & 8 & 12 & 24 \\
\hline MM2 & 0 & 1 & 2 & 4 & 7 & 30 \\
\hline \multicolumn{5}{|c|}{ Teste - Fentanil, etomidato, remifentanil, propofol } & (1) & \\
\hline MMP & 0 & 0 & o & 1 & 1 & 29 \\
\hline MM1 & 0 & o & 0 & 1 & 1 & 29 \\
\hline MM2 & 0 & o & 0 & 1 & 1 & 30 \\
\hline \multicolumn{5}{|c|}{ Teste - Remifentanil, propofol } & (2) & \\
\hline MMP & 0 & 0 & 1 & 1 & 2 & 25,5 \\
\hline MM1 & 0 & 0 & 1 & 1 & 2 & 25 \\
\hline MM2 & 0 & o & o & 1 & 1 & 29 \\
\hline \multicolumn{5}{|c|}{ Teste - Fentanil, propofol } & (4) & \\
\hline MMP & 0 & 0 & 3 & 1 & 4 & 23,75 \\
\hline MM1 & o & o & 3 & 1 & 4 & 23 \\
\hline MM2 & 0 & 0 & 0 & 1 & 1 & 23 \\
\hline \multicolumn{5}{|r|}{ ntes } & 28 & \\
\hline
\end{tabular}

A para a variável (entre parênteses) e em cada fase do teste; 'Todos os pacientes que não responderam no MM1 estavam intubados, portanto, incapazes de responder o questionário; "Todos os pacientes que não responderam no MM2 tiveram alta antes da terceira reaplicação.

\section{DISCUSSÃO}

Neste estudo avaliamos o DPAO em uma amostra de pacientes idosos atendidos pelo SUS em um hospital universitário de médio porte no sul de Minas Gerais, Brasil, que oferece uma grande variedade de procedimentos cirúrgicos. Observou-se um perfil heterogêneo entre os avaliados, relativo à escolaridade e faixa etária, bem como para as condições clínicas que direcionaram os protocolos analgésico-anestésicos (doses, profundidade) nas diferentes cirurgias realizadas. Tais fatores podem influenciar nos desfechos relativos à prevalência de DPAO, embora não houve para este estudo correlação significativa entre algumas variáveis ${ }^{12-17}$.

O MEEM foi adotado para avaliar o DPAO, baseado em critérios de estratificação e interpretação propostos por Brucki et al. ${ }^{11}$. O uso do MEEM para estabelecer DPAO levanta discussões a cerca da padronização para o Brasil e a falibilidade das versões adaptadas do teste original. Tendo como base principalmente o nível de escolaridade (anos de estudo) para a interpretação dos scores, algumas especificidades da população avaliada podem ser negligenciadas no MEEM, abrindo espaço para controvérsias em relação ao exame ${ }^{18}$.

Avaliando individualmente o MEEM, detectou-se neste estudo uma significativa prevalência de DPAO - 21,4\% $(n=6)$, com $14,3 \%(n=4)$ dos casos ocorrendo em D1, $3,6 \%(n=1)$ em D3 e 3,6\% $(n=1)$ em ambos os períodos. Embora as cirurgias cardíacas tenham sido as mais realizadas neste estudo, a DPAO foi mais prevalente em pacientes submetidos a tais cirurgias, corroborando prévios estudos que apontam esta tendência de acordo com a topografia cirúrgica e fatores relacionados (como uso de sondas, comorbidades, entre outros), bem como destacam as doenças cardíacas como um fator predisponente para o DPAO $^{12,19,20 .}$.

No presente estudo, não foi identificada relação significativa entre 0 nível de escolaridade geral e a pontuação no MEEM nos momentos D0, D1 e D3, ainda que o nível de escolaridade seja um fator que influência nos resultados do teste ${ }^{18,21}$, bem como o padrão de estratificação para os níveis de pontuação. Contudo, adotando-se a metodologia da versão de 1980 do MEEM, a ocorrência de possíveis estados de DPAO seria diferente, como sugere as médias da pontuação dos homens e mulheres voluntários aqui avaliados. Machado et al. $^{21}$ mostraram uma diferença significativa nos resultados do MEEM em relação ao nível de escolaridade, com média de 19,8 pontos para os detentores de escolaridade de 4-9 anos, e 24,5 para aqueles com mais de nove anos de escolaridade, na versão do teste utilizada no estudo. Frente a isso, existe uma necessidade de mais estudos complementares que objetivem uma melhoria das adaptações do MEEM, inclusive com versões locais mais apropriadas, considerando toda a heterogeneidade da população brasileira e distintas variáveis que venham interferir nos resultados do teste, como os fatores predisponentes para a DPAO apontados por Bisinotto et al. ${ }^{12}$. 
De nota, houve uma diferença significativa na pontuação do MEEM relacionada ao sexo do paciente. Tal observação demanda estudos mais aprofundados, com um número maior de voluntários e equiparidade entre o número de homens e mulheres no estudo ${ }^{21,22}$. Assim, no presente estudo não observamos a influência da escolaridade e tipos de protocolos anestésicocirúrgicos sobre a pontuação, fatores que são considerados principais interferentes na interpretação do MEEM, mas o presente estudo demonstra que o sexo pode ser mais um fator para a interpretação estratificada do teste, desde que apenas esta variável influenciou a pontuação média dos entrevistados, servindo como base para futuras considerações sobre as dificuldades de padronização e interpretação do MEEM.

Com relação às condições clínicas, não houve diferença significativa entre a pontuação nos testes aplicados no pré e pós-operatório e a utilização de anestésico geral, o que talvez possa ser devido à possibilidade de aprendizado do teste pelos voluntários do estudo. Contudo, o protocolo fentanil/propofol/etomidato, usado para CRVM e CCV, foi 0 adotado em pacientes que apresentaram os melhores resultados no MEEM, uma vez que houve os maiores valores em M2 e, em indivíduos com apenas ensino fundamental. Machado et al. ${ }^{21}$ sugerem a ocorrência de memorização das respostas e do raciocínio pelos pacientes devido ao curto espaço entre as reaplicações do teste, e faz ressalva para a não comunicação ao voluntário da repetição do teste, objetivando evitar o estímulo ao aprendizado.

No contexto deste estudo, os fatores de risco para a DPAO devem também ser levados em conta, visando à implementação de medidas preventivas para a promoção de saúde do paciente cirúrgico, e na tentativa de minimizar a morbidade e mortalidade associadas a esta condição, desde que as opções terapêuticas para o DPAO são limitadas ${ }^{12,23,24}$.

CONCLUSÃO

Houve uma significativa prevalência de DPAO na amostra de idosos submetidos a diferentes tipos de cirurgias e protocolos usando diferentes AGs. Uma maior pontuação média no MEEM ocorreu para o sexo masculino, mas não se observou diferença significativa entre o nível de escolaridade, faixa etária, tipo de cirurgia ou uso de AGs e a pontuação no teste. Alguns vieses tais como heterogeneidade dos protocolos cirúrgico-anestésicos, idade, sexo e escolaridade, bem como fatores de risco para a DPAO devem ser considerados e a interpretação do MEEM deve ser cautelosa. AGRADECIMENTOS

O presente trabalho foi realizado com apoio da Coordenação de Aperfeiçoamento de Pessoal de Nível Superior - Brasil (CAPES) Código de Financiamento 001.

REFERÊNCIAS

1. Cangiani M. Medicina Peri operatória, anestesia ambulatorial em paciente idoso. Rev Bras Anestesiol. 2000;50:68-85.

2. de Paula TC, Bochner R, Montilla DER. Análise clínica e epidemiológica das internações hospitalares de idosos decorrentes de intoxicações e efeitos adversos de medicamentos, Brasil, de 2004 a 2008. Rev Bras Epidemiol. 2012;15(4):828-44.

3. Araujo PL, Galato D. Risco de fragilização e uso de medicamentos em idosos residentes em uma localidade do sul de Santa Catarina. Rev Bras Geriatr Gerontol. 2012;15(1):119-26.

4. Liu $X, Y u Y$, Zhu S. Inflammatory markers in postoperative delirium (POD) and cognitive dysfunction (POCD): A meta-analysis of observational studies. PLoSONE. 2018;13(4): e0195659.

5. Hudetz JÁ, lqbal Z, Gandhi SD, et al. Postoperative cognitive dysfunction in older patients with a history of alcohol abuse. Anesthesiol. 2007;106:423-30.

6. Rasmussen LS, Johnson T, Kuipers HM, et al. Does anaesthesia cause postoperative cognitive dysfunction? A randomized study of regional versus general anaesthesia in 438 elderly patients. Acta Anaesthesiol Scand. 2003;47:260-66.

7. Anwer HMF, Swelem SE, El-Sheshai A, et al. Postoperative cognitive dysfunction in adult and elderly patients. Middle East J Anesth. 2006; 18:1123-38.

8. Janjua MS, Arthur ME. Postoperative Delirium. NCBI Bookshelf: StatPearls Publishing;2019.

9. Praticò C, Quattrone D, Lucanto T, et al. Drugs of anestesia acting on central cholinergic system may cause post-operative cognitive dysfunction and delirium. Med Hypotheses. 2005 65(5):972-82.

10. Afonso J, Reis F. Dexmedetomidina: papel atual em anestesia e cuidados intensivos. Rev Bras Anestesiol, 2012;62(1):125-33.

11. Brucki S, Nitrini R, Caramelli $P$, et al. Sugestões para o uso do mini-exame do estado mental no Brasil. Arq Neuropsiquiatr. 2003; 61(3-B):777-81.

12. Bisinotto FMB, Silveira LAM, Silva RO, et al. Delirium pós-operatório no idoso. Onde estamos? Rev Med Minas Gerais. 2017; 27(Supl 2):S52-S66. 
13. Djokovic JL. Prediction of outcome of surgery and anesthesia in patients over 80. JAMA. 1979;242(21):2301-6.

14. Bryson GL, Wyand A. Evidence-based clinical update: General anesthesia and the risk of delirium and post-operative cognitive dysfunction. Can J Anaesth. 2006;53:669-77.

15. Dasgupta M, Dumbrell AC. Preoperative risk assessment for delirium after noncardiac surgery: A systematic review. J Am Geriatr Soc. 2006; 54(10):1578-89.

16. Papaioannou A, Fraidakis $O$, Michaloudis D, et al. The impact of the type of anaesthesia on cognitive status and delirium during the first postoperative days in elderly patients. Eur $\mathrm{J}$ Anaesthesiol. 2005;22(7):492-99.

17. Orena EF, King AB, Hughes CG. The role of anesthesia in the prevention of postoperative delirium: a systematic review. Minerva Anestesiol. 2016;82(6):669-83.

18. Melo DM, Barbosa AJG. O uso do Mini-Exame do Estado Mental em pesquisas com idosos no Brasil: uma revisão sistemática. Ciênc saúde colet. 2016;20(12):3865-76.

19. Silverstein JH, Timberger $M$, Reich $\mathrm{DL}$, et al. Central nervous system dysfunction after noncardiac surgery and anestesia in the elderly. Anesthesiol. 2007;106:622-28.

20. Croughwell ND, Newman MF, Blumenthal JA, et al. Jugular bulb saturation and cognitive dysfunction after cardiopulmonary bypass. Ann Thorac Surg. 1994; 58:1702-8.

21. Machado $H$, Pereira MJB, Orfão $J$, et al. Changes in cognitive performance in patients submitted to anesthesia. Acta méd port. 2000; 13(3):85-92.

22. Boos GL, Soares LF, De Oliveira Filho GL. Disfunção Cognitiva Pós-Operatória: Prevalência e Fatores Associados. Rev Bras Anestesiol. 2005;55(5):517-24.

23. Kehlet H, Dahl JB. Anaesthesia, surgery, and challenges in postoperative recovery. Lancet. 2003; 362(9399): 1921-1928.

24. Robinson TN, Raeburn CD, Tran ZV, et al. Postoperative delirium in the elderly: risk factors and outcomes. Ann Surg. 2009;249(1):173-8.

\section{CONFLITO DE INTERESSES}

Os autores declaram não haver conflitos de interesse

AUTOR PARA CORRESPONDÊNCIA

\section{Dr. Cláudio Daniel Cerdeira}

DBq, ICB, UNIFAL-MG,

Rua Gabriel Monteiro da Silva, 700, Prédio E, S 207 C, 37130-001 Alfenas - MG, Brasil

E-mail: daniel.cerdeira.84@gmail.com

Submetido em 24/11/2020

Aceito em 02/08/2021 